\title{
Kinematic Control and Obstacle Avoidance for Soft Inflatable Manipulator ${ }^{\star}$
}

\author{
Ahmad Ataka ${ }^{1,2}$, Agostino Stilli ${ }^{3}$, Jelizaveta Konstantinova ${ }^{2}$, Helge A \\ Wurdemann $^{4}$, and Kaspar Althoefer ${ }^{2}$ \\ 1 The Centre for Robotics Research (CoRe), Department of Informatics, Kings \\ College London, London WC2R 2LS, United Kingdom. \\ 2 The Centre for Advanced Robotics @ Queen Mary (ARQ), Faculty of Science and \\ Engineering, Queen Mary University of London, Mile End Road, London E1 4NS, \\ United Kingdom. \\ 3 The Dept. of Computer Science, University College London, London WC1E 7JE, \\ United Kingdom. \\ 4 The Dept. of Mechanical Engineering, University College London, London WC1E \\ 7JE, United Kingdom. ahmad_ataka_awwalur.rizqi@kcl.ac.uk
}

\begin{abstract}
In this paper, we present a kinematic control and obstacle avoidance for the soft inflatable manipulator which combines pressure and tendons as an actuating mechanism. The position control and obstacle avoidance took inspiration from the phenomena of a magnetic field in nature. The redundancy in the manipulator combined with a planar mobile base is exploited to help the actuators stay under their maximum capability. The navigation algorithm is shown to outperform the potential-field-based navigation in its ability to smoothly and reactively avoid obstacles and reach the goal in simulation scenarios.
\end{abstract}

Keywords: Kinematic Control · Obstacle Avoidance · Soft Manipulator - Bio-inspired Robot.

\section{Introduction}

Soft robotics has been at the forefront of the robotics revolution in the last decade. Robots with soft and flexible materials, often taking inspiration from properties of biological organisms in nature, have been developed to handle tasks previously untouched by the rigid robots counterparts. These include minimally invasive surgery [7], whole-body grasping, and manoeuvring in tight space surrounded by cluttered environment [16]. The emergence of smart materials and low-cost fabrication techniques have also led to various designs and actuating mechanisms, which include pneumatically-actuated systems [8], tendon-driven systems [15], or a combined antagonistically tendon-pressure actuation system

\footnotetext{
* This work was supported in part by King's College London, the EPSRC in the framework of the NCNR (National Centre for Nuclear Robotics) project (EP/R02572X/1), q-bot led project WormBot (2308/104059), and the Indonesia Endowment Fund for Education, Ministry of Finance Republic of Indonesia.
} 
[12]. Despite all of these promising developments, new challenges arise in the field of robot modelling [17], control [14], and navigation [4], mainly due to the non-linear behaviour of the flexible structure employed.

One of the emerging technologies in the field of soft robotics is the use of an inflatable structure which enables the robot to grow over a wide range of length [11]. This type of robot is useful not only due to the compliance of the material employed but also due to its ability to grow from a tiny size to a very long structure. Current applications include an antenna reconfiguration [5] and systems that can be inserted through a narrow opening used in a laparoscopic surgery [12]. The inflatability of the structure makes this type of robot a prime candidate for further applications where cluttered environments are present, such as the nuclear industry or a below-ground environment. However, research on control and navigation of this type of robot is still in its infancy.

For soft robots in general, the majority of the navigation methods employed in the literature rely on either an optimization technique [10], [13] or samplingbased planners [19], [21]. While practically useful even in complex environments, these types of methods rely on the availability of a complete or at least near-complete knowledge of the environment. Recent effort which implemented a neural dynamics approach [6] poses a similar complication, while another method which used a supervised learning-by-demonstration [20] relies on human interventions for training. On the other hand, the use of reactive obstacle avoidance, such as presented in [9], [3], is very susceptible to a local minima problem, a scenario where the robot gets stuck in a configuration before reaching the target. Besides, none of these works deals with the inflatability of the robotic structure which is the main characteristics of the soft inflatable manipulator in this paper.

In this paper, we present a kinematic control and obstacle avoidance for the soft inflatable manipulator based on the design presented in [18]. A beamtheory-based kinematic model based on the previous work in [18] is used. The position control and obstacle avoidance are based on our previous work in [4] where we applied reactive magnetic-field-inspired navigation to multi-segments soft continuum manipulator. Our contribution is twofold:

1. we apply the magnetic-field-inspired navigation as a way to avoid unknown obstacle reactively for the soft inflatable arm, and

2. we exploit the redundancy on the beam-theory kinematic model to help the robot avoiding the actuators' constraints when navigating the environment.

\section{Kinematic Modelling}

\subsection{Design of the inflatable manipulator}

The design of the soft inflatable robotic manipulator is based on the previous works described in [18]. The manipulator consists of a hyperelastic latex bladder whose endpoint is attached to an inextensible fabric. A hollow tube is connected to the bladder as a means for air to be pumped in. The outer fabric is equipped with three channels where each channel is radially separated by $120^{\circ}$ from the 


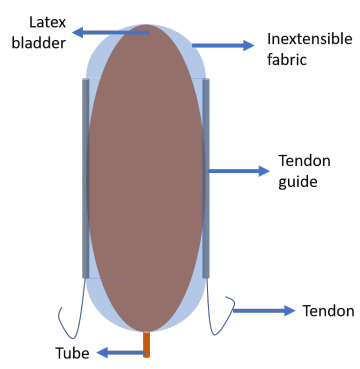

(a)

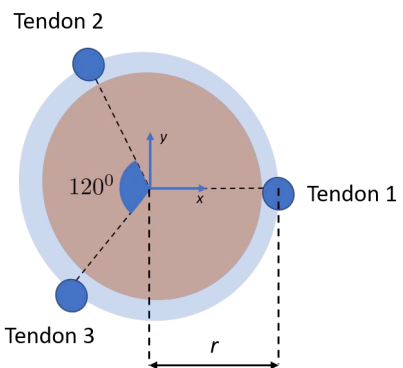

(b)

Fig. 1. The mechanical design of the soft inflatable manipulator.
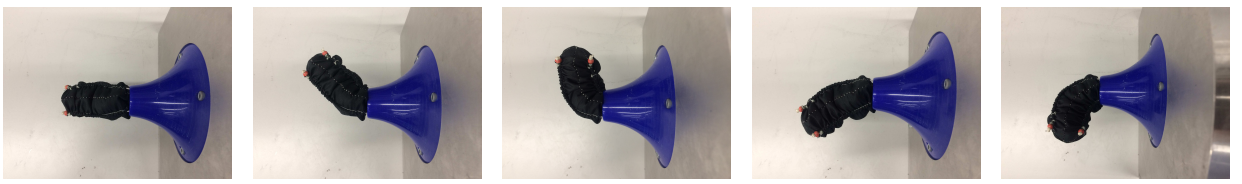

Fig. 2. The manoeuvrability of the soft, inflatable manipulator.

other two channels. Three nylon tendons are guided along these channels and fixed at the distal end of the manipulator. The mechanical design of the manipulator is illustrated in Fig. 1. Air can be pumped in through the hollow tube into the bladder to change the state of the manipulator from a deflated state to an inflated state. Since the outer fabric is unstretchable, the radial expansion of the elastic bladder will be constrained up to the cross-sectional area of the outer fabric. This constraint forces the bladder to expand only in the direction of the robot's longitudinal axis, i.e. causing the robot structure to elongate. Once fully inflated, further variations of the air pressure inside the bladder will result in variations of the manipulator's structural stiffness.

Besides the air pressure inside the bladder, length variations of the three tendons will also affect the manipulator's shape. These enable the manipulator to perform not only elongation but also bending in a 3-dimensional environment as shown in Fig. 2. Any difference in the tendons' length will lead to a 3-dimensional bending. The overall combination of the tendons' length and the air pressure will move the manipulator's tip to a particular position. A stepper motor is connected to each tendon via a linear actuator used to control the tension of the tendons. A linear pressure regulator is used for regulating the pressure from an air compressor to manipulate the air pressure inside the latex bladder.

\subsection{Beam kinematic model}

As can be observed in Fig. 2, the segment of the manipulator can be modeled as a circular arc with a constant curvature. Hence, the shape of the segment can 
be parameterized by a set of configuration space variables $\mathbf{k}=[\kappa \phi l]^{T}$ which represent a curvature $\kappa$, a rotational deflection angle $\phi$, and a segment length $l$. The actuator space variables $\mathbf{q}_{A}=\left[f_{1} f_{2} f_{3} p\right]^{T}$ consist of an air pressure $p$ and force $f_{i}$ of tendon- $i$. The workspace variable $\mathbf{x} \in \mathbb{R}^{3}$ stands for the tip position. The kinematic model maps the actuator space variables $\mathbf{q}_{A}$ to the configuration space variables $\mathbf{k}=h\left(\mathbf{q}_{A}\right)$ and finally to the workspace variables $\mathbf{x}=g(\mathbf{k})$.

The kinematic model of the inflatable arm is based on the Euler-Bernoulli beam theory as has been described in [18]. As shown in Fig. 1b, the tension $f_{i}$ of each tendon produces a bending moment $\mathbf{M}$ given by

$$
\mathbf{M}=\sum_{i=1}^{3} \mathbf{r}_{i} \times \mathbf{F}_{i} .
$$

$\mathbf{r}_{i}$ stands for the vector position of tendon- $i$ with respect to the manipulator's central axis. From Fig. 1b, it can be expressed as $\mathbf{r}_{i}=\left[\begin{array}{lll}r \cos \left(\frac{2 \pi}{3} i\right) & r \sin \left(\frac{2 \pi}{3} i\right) & 0\end{array}\right]^{T}$, where $r$ stands for the cross-sectional radius. $\mathbf{F}_{i}$ stands for the tension vector of tendon- $i$ and is defined as $\mathbf{F}_{i}=\left[\begin{array}{lll}0 & 0 & f_{i}\end{array}\right]^{T}$. The magnitude of the bending moment is proportional to the curvature $\kappa$ of the manipulator segment as follows

$$
\kappa=\frac{|\mathbf{M}|}{E I},
$$

where $E$ stands for the Young modulus of the material while $I$ stands for the cross-sectional moment of inertia which is defined as $I=\frac{\pi}{2} r^{4}$ for a circular cross section with radius $r$. The bending moment $\mathbf{M}$ also produces a rotational deflection angle $\phi$. We can derive the value of $\phi$ as follows

$$
\phi=\arctan 2\left(-M_{x}, M_{y}\right) .
$$

The tension on the tendons and the pressure of the elastic bladder will contribute to the increase of length $l$. The total force acting on the manipulator is given by

$$
F_{\text {tot }}=\left(p-p_{0}\right) A-\sum_{i=1}^{3} f_{i}
$$

where $A=\pi r^{2}$ stands for the cross-sectional area of the manipulator and $p_{0}$ stands for the initial pressure. The increase in the manipulator's length is

$$
\Delta l=l-l_{0}=\frac{F_{t o t}}{E A} l_{0},
$$

where $l_{0}$ stands for the initial length.

Eq. (1)-(5) map the actuator space variables $\mathbf{q}_{A}$ to the configuration variables $\mathbf{k}$. To get the workspace variables $\mathbf{x}$, we use the geometrical property of a circular arc. The homogeneous transformation matrix describing the tip pose of the manipulator with respect to the base, $\mathbf{T}(\mathbf{k}) \in S E(3)$, is described in [17]. The tip position $\mathbf{x} \in \mathbb{R}^{3}$ can be expressed as

$$
\mathbf{x}=\left[\frac{1}{\kappa} \cos \phi(1-\cos \kappa l) \frac{1}{\kappa} \sin \phi(1-\cos \kappa l) \frac{1}{\kappa} \sin \kappa l\right]^{T} .
$$




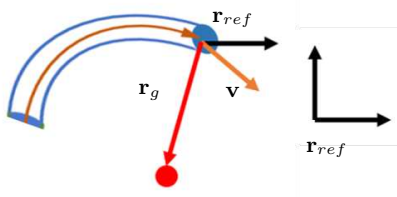

(a)

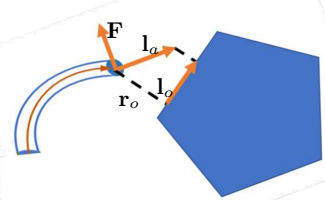

(b)

Fig. 3. (a) The tip of the manipulator moves with velocity $\mathbf{v}$ and located at $\mathbf{r}_{g}$ from the goal. $\mathbf{r}_{r e f}$ is a static reference vector. (b) The tip of the manipulator generates an artificial current $\mathbf{l}_{o}$ on the obstacle surface.

In this paper, we assume that the soft inflatable manipulator is put on top of a mobile platform which is able to move translationally in a planar environment. In this case, the pose of the tip with respect to a static world frame $\mathbb{W}$ is described as ${ }^{\mathbb{W}} \mathbf{T}(\mathbf{q})=\mathbf{T}_{B}\left(\mathbf{q}_{B}\right) \mathbf{T}\left(\mathbf{q}_{A}\right)$, where $\mathbf{T}_{B} \in S E(3)$ stands for a homogeneous transformation matrix of the frame attached to the base with 2 translational degrees of freedom and $\mathbf{q}_{B} \in \mathbb{R}^{2}$ stands for the position of the base. Hence, the overall actuator space variables are described by $\mathbf{q}=\left[\mathbf{q}_{B} \mathbf{q}_{A}\right]^{T}$. From the kinematic model, we can also derive the Jacobian matrix defined as $\mathbf{J}(\mathbf{q}) \in$ $\mathbb{R}^{3 \times 6}=\frac{\partial \mathbf{x}}{\partial \mathbf{q}}$ using a numerical computation as described in [4].

\section{Tip Navigation and Obstacle Avoidance}

\subsection{Go-to-goal Navigation}

The geometric control approach, as presented in [4], is used to navigate the tip of the arm towards the desired position. This approach is chosen since it can maintain the manipulator's tip to move at an almost constant speed during the movement. By setting the speed to be small, we can ensure that the motion of the manipulator is slow enough such that its dynamics can be neglected.

Suppose that the manipulator's tip moves with velocity $\mathbf{v}$ as illustrated in Fig. 3a. We can express the direction of velocity vector $\mathbf{v}$ as a rotation matrix with respect to a static unit reference vector $\mathbf{r}_{r e f}$ as $\mathbf{R}_{v}=\mathbf{I}+\hat{\omega}_{v}+\hat{\omega}_{v}^{2} \frac{1}{1+\cos \phi}$. Here, $\omega_{v}=\hat{\mathbf{r}}_{r e f} \frac{\mathbf{v}}{|\mathbf{v}|}, \cos \phi=\mathbf{r}_{r e f}^{T} \frac{\mathbf{v}}{|\mathbf{v}|}$, while $\hat{\mathbf{l}}$ is defined as a skew-symmetric matrix of a vector $\mathbf{l}$ as follows

$$
\hat{\mathbf{i}}=\left[\begin{array}{ccc}
0 & -l_{z} & l_{y} \\
l_{z} & 0 & -l_{x} \\
-l_{y} & l_{x} & 0
\end{array}\right] .
$$

$\mathbf{I} \in \mathbb{R}^{3 \times 3}$ is an $3 \times 3$ identity matrix. We can use the same procedure for vector $\mathbf{r}_{g}=\mathbf{x}_{\mathbf{g}}-\mathbf{x}$ to get the direction of the goal $\mathbf{R}_{g}$ with respect to frame $\mathbf{r}_{r e f}$.

The aim is to rotate the direction of the manipulator's tip from arbitrary orientation $\mathbf{R}_{v}$ to the goal orientation $\mathbf{R}_{g}$. The control law is described as follows

$$
\hat{\omega}_{g}=-K_{\omega} \log \left(\mathbf{R}_{e}\right),
$$


where $\mathbf{R}_{e}$ stands for an error matrix and is defined as $\mathbf{R}_{e}=\mathbf{R}_{g}^{T} \mathbf{R}_{v}$. Here, $K_{\omega} \geq 0$ is a constant while the operator $\log (\mathbf{R})$ for any $\mathbf{R} \in S O(3)$ is defined as $\log (\mathbf{R})=\frac{\beta}{2 \sin \beta}\left(\mathbf{R}-\mathbf{R}^{T}\right)$ where $\beta=\arccos \left(\frac{\operatorname{tr}(\mathbf{R})-1}{2}\right)$. The angular speed $\omega_{g} \in \mathbb{R}^{3}$ expressed in the goal frame $\mathbf{R}_{g}$ can be retrieved from $\hat{\omega}_{g}$ in (8) by exploiting the skew-symmetric matrix definition in (7). To get the angular speed $\omega_{r e f}$ in the static frame $\mathbf{r}_{r e f}$, a matrix transformation is used as follows $\omega_{r e f}=\mathbf{R}_{g} \omega_{g}$. Lastly, the force needed to generate this required angular speed is given by

$$
\mathbf{F}_{g c}=\hat{\omega}_{r e f} \mathbf{v} .
$$

To generate the initial movement, an additional controller is used as follows

$$
\mathbf{F}_{v}=-K_{v}\left(v-v_{d}\right) \mathbf{d},
$$

in which $K_{v}>0$ represents a positive constant, $v_{d}$ denotes the desired speed of the manipulator's tip, while $\mathbf{d}$ is defined as $\mathbf{d}=\left\{\begin{array}{ll}\frac{\mathbf{r}_{g}}{\left|\mathbf{r}_{g}\right|} & \text { if }|\mathbf{v}|=0 \\ \frac{\mathbf{v}}{v} & \text { if }|\mathbf{v}|>0\end{array}\right.$. Similarly, in order to force the manipulator's tip to asymptotically stop at the goal position, a proportional-derivative controller $\mathbf{F}_{p d}$ is employed when the robot is located at a distance smaller than a limit distance $r_{g l}$ to the goal. Finally, the overall control signal to govern the tip's movement to the goal is described as follows

$$
\mathbf{F}_{t g}=\left\{\begin{array}{ll}
\mathbf{F}_{g c}+\mathbf{F}_{v} & \text { if }\left|\mathbf{r}_{g}\right| \geq r_{g l} \\
\mathbf{F}_{p d} & \text { if }\left|\mathbf{r}_{g}\right|<r_{g l}
\end{array} .\right.
$$

\subsection{Magnetic-field-inspired Obstacle Avoidance}

To achieve obstacle avoidance, the magnetic-field-inspired obstacle avoidance based on the previous works described in [1], [2], [4], is employed. The obstacle avoidance algorithm is inspired by a phenomenon observed when a charged particle moves close to a current-carrying wire. A moving robot (in this case the tip of a soft inflatable manipulator) will induce an artificial current on the closest obstacle surface. The artificial current, in parallel to the real electric current in a wire, produces a magnetic field which affects the movement of the robot in such a way that the robot will avoid collision with the obstacle.

The tip induces an electric current $\mathbf{l}_{o}$ on the closest obstacle described by

$$
\mathbf{l}_{o}=\mathbf{l}_{a}-\frac{\left(\mathbf{l}_{a}^{T} \mathbf{r}_{o}\right) \mathbf{r}_{o}}{\left|\mathbf{r}_{o}\right|^{2}} .
$$

Here, $\mathbf{l}_{a}$ and $\mathbf{r}_{o}$ denote a unit vector of the tip's movement direction and the closest obstacle position with respect to the robot's tip, respectively. From the geometrical perspective, eq. (12) defines the artificial current $\boldsymbol{l}_{o}$ as a projection of the tip's velocity direction $\mathbf{l}_{a}$ on to the obstacle surface as illustrated in Fig. $3 \mathrm{~b}$. To avoid collisions, the manipulator's tip needs to follow the direction of artificial current $\mathbf{l}_{o}$. To ensure this behaviour, the vector field $\mathbf{F}_{o}$ is defined as

$$
\mathbf{F}_{o}=c \mathbf{l}_{a} \times\left(\mathbf{l}_{o} \times \mathbf{l}_{a}\right) f\left(r_{o}, v\right),
$$




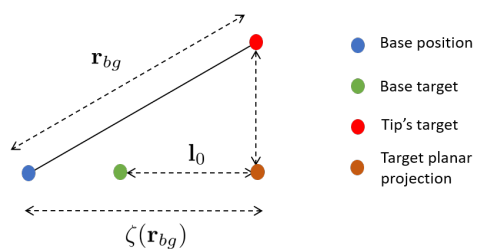

(a)

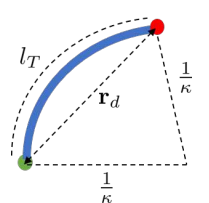

(b)

Fig. 4. (a) The base (blue dot) will be forced to move to a target position (green dot) located at a distance $l_{0}$ from the projection (orange dot) of the goal position (red dot). (b) The length of the manipulator will be forced to reach a target length $l_{T}$, i.e. the curve length from the target base position (green dot) to the goal position (red dot).

where $c>0$ denotes a constant, $f\left(r_{o}, v\right) \geq 0$ denotes a positive scalar function, and $v$ denotes the speed of the manipulator's tip. To ensure collision avoidance with the obstacle surface, we set the scalar function $f\left(r_{o}, v\right)$ to be proportional to the speed of the manipulator's tip $v$ and inversely proportional to the distance towards the obstacle surface $r_{o}$ once the distance between the tip and the closest obstacle is smaller than a limit distance $r_{l}$ as follows $f\left(r_{o}, v\right)=\left\{\begin{array}{ll}\frac{v}{r_{o}} & \text { if } r_{o}<r_{l} \\ 0 & \text { if } r_{o} \geq r_{l}\end{array}\right.$.

\subsection{Implementation Strategy}

It is not possible to directly apply the proposed vector field to the model of the manipulator described in Section 2.2 since it describes only the kinematics, not dynamics of the system. To apply the proposed vector field to the kinematic model without losing its properties, the force $\mathbf{F}_{t}$ is numerically integrated to generate a task-space velocity $\dot{\mathbf{x}}$ as follows $\dot{\mathbf{x}}(t+\Delta t)=\dot{\mathbf{x}}(t)+\mathbf{F}_{t}(t) \Delta t$, where $\Delta t$ stands for a time-integration constant.

For the case of a mobile base, we have a redundant system where $\mathbf{J}(\mathbf{q}) \in \mathbb{R}^{3 \times 6}$. This redundancy can be exploited to ensure that the manipulator's tip can reach the desired target and, at the same time, the manipulator's actuators stay inside their actuating capability. The actuator space velocity $\dot{\mathbf{q}}$ is given by [4]

$$
\dot{\mathbf{q}}=\mathbf{J}^{+} \dot{\mathbf{x}}+\lambda\left(\mathbf{I}-\mathbf{J}^{+} \mathbf{J}\right) \mathbf{z},
$$

where $\lambda$ stands for a positive constant, ${ }^{+}$stands for a pseudo-inverse operation described by $\mathbf{J}^{+}=\mathbf{J}^{T}\left(\mathbf{J J}^{T}\right)^{-1}$, and $\mathbf{I} \in \mathbb{R}^{6 \times 6}$ is an identity matrix. The vector $\mathbf{z} \in \mathbb{R}^{6}$ stands for an arbitrary vector which will be chosen to ensure an actuator constraint avoidance. Each actuator has a target value to be achieved.

For the base, the target position is given by

$$
\mathbf{x}_{B T}=l_{0} \frac{\zeta\left(\mathbf{r}_{b g}\right)}{\left|\zeta\left(\mathbf{r}_{b g}\right)\right|}+\zeta\left(\mathbf{x}_{\mathbf{g}}\right)
$$

where $l_{0}$ stands for an initial relaxed length of the manipulator, $\mathbf{r}_{b g}=\mathbf{x}_{b}-\mathbf{x}_{\mathbf{g}}$ stands for a relative position between the base position $\mathbf{x}_{b}$ and the goal position 
Table 1. A list of parameters.

\begin{tabular}{|l|l|l|l|l|l|}
\hline Parameter & Value & Parameter & Value & Parameter & Value \\
\hline$r$ & $0.015 \mathrm{~m}$ & $E$ & $60000 \mathrm{~Pa}$ & $p_{0}$ & $100000 \mathrm{~Pa}$ \\
$l_{0}$ & $0.09 \mathrm{~m}$ & $K_{\omega}$ & 10 & $K_{v}$ & 50 \\
$c$ & 1.5 & $\Delta t$ & $0.02 \mathrm{~m}$ & $v_{d}$ & $0.025 \mathrm{~m} / \mathrm{s}$ \\
$K_{P z}$ & 6 & $K_{D z}$ & 1 & $\lambda$ & 1 \\
$r_{g l}$ & $0.05 \mathrm{~m}$ & $f_{\min }$ & $0 \mathrm{~N}$ & $f_{\max }$ & $5 \mathrm{~N}$ \\
\hline
\end{tabular}

$\mathbf{x}_{\mathbf{g}}$, while $\zeta(\mathbf{x})$ is an operation which returns a planar version of a vector $\mathbf{x}$. This base target position will help the pressure avoiding its actuating constraint since it will bring the mobile base closer to the goal position. More specifically, eq. (15) tries to keep the base located at a planar distance which is equal to the normal length $l_{0}$ from the goal position as illustrated in Fig. 4a.

For the tendons, the target value is its mid-value $f_{T}$ between its minimum $f_{\min }$ and maximum tensions $f_{\max }$. For the pressure, the target value is

$$
p_{T}=p_{0}+\frac{\sum_{i=1}^{3} f_{i}}{A}+\frac{l_{T}-l_{0}}{l_{0}} E .
$$

Slotting (16) into (4)-(5), we can see that this target pressure will force the manipulator's length $l$ to be $l_{T}$. Here, we choose this target length $l_{T}$ as follows

$$
l_{T}=\left\{\begin{array}{ll}
\left.\frac{2}{\kappa} \arcsin \frac{\left(\kappa\left|\mathbf{r}_{d}\right|\right.}{2}\right) & \text { if } \kappa \neq 0 \\
\left|\mathbf{r}_{d}\right| & \text { if } \kappa=0
\end{array},\right.
$$

where $\mathbf{r}_{d}=\mathbf{r}_{\mathbf{g}}-\mathbf{x}_{B T}$. This target length $l_{T}$ is the length of a circular arc which spans from the target base position $\mathbf{x}_{B T}$ to the goal position $\mathbf{r}_{\mathbf{g}}$. Hence, this will make the manipulator easier to achieve the target base position $\mathbf{x}_{B T}$ while at the same time allowing the tip to reach the goal as illustrated in Fig. 4b.

Finally, to achieve these targets, the rate of vector $\mathbf{z}$ is defined as

$$
\dot{\mathbf{z}}=\left(-K_{P z}\left(\mathbf{q}-\mathbf{q}_{T}\right)-K_{D z} \dot{\mathbf{q}}\right)
$$

where $K_{P z}$ and $K_{D z}$ stand for positive constants while the target vector $\mathbf{q}_{T}$ is defined as $\mathbf{q}_{T}=\left[\begin{array}{lll}\mathbf{x}_{B T} & f_{T} \mathbf{I}_{3} p_{T}\end{array}\right]^{T}$. Here, $\mathbf{I}_{3} \in \mathbb{R}^{3}$ stands for an identity vector. Finally, vector $\mathbf{z}$ is given by $\mathbf{z}(t+\Delta t)=\dot{\mathbf{q}}(t)+\dot{\mathbf{z}} \Delta t$.

\section{Results and Analysis}

In this section, the performance of the proposed algorithm is evaluated in the simulation scenario. The parameters used for the manipulator's modelling and the navigation algorithm are presented in Table 1. In this paper, we consider only static obstacles in the environment whose shape and position are unknown to the manipulator before the movement execution. We assume that the manipulator 

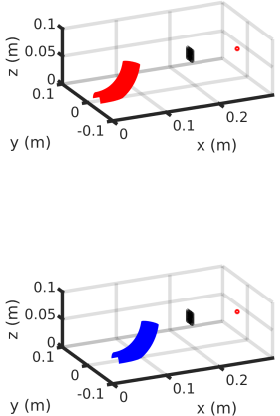

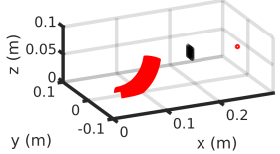

(a)

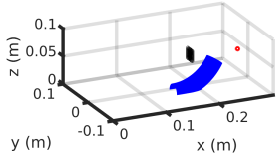

(b)
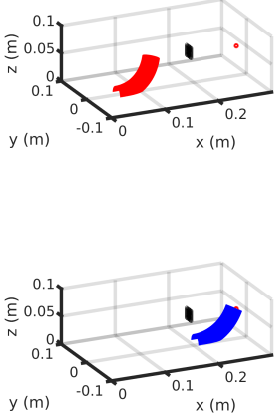

Fig. 5. The soft inflatable arm with a mobile base navigates an environment consisting of a planar obstacles with (a) an electric-field-potential algorithm and (b) a magneticfield-inspired algorithm. The order of movement is from left to right.

is equipped with a sensor which is able to detect the surrounding obstacles as far as a distance $r_{l}=0.04 \mathrm{~m}$. In some of the scenarios, we compare the performance of the algorithm with the electric-field-based navigation as described in [3].

The first environment consists of a single planar obstacle (drawn in black) where the manipulator's tip needs to reach the desired position (shown as a red dot) across the obstacle as shown in Fig. 5. We compare the performance of the electric-field-potential algorithm (Fig. 5a) with the proposed magnetic-fieldinspired algorithm (Fig. 5b) when applied to the model of the soft inflatable manipulator. We can see how the electric-field-potential algorithm fails to guide the tip of the manipulator to reach the target in Fig. 5a. This is due to the repulsive nature of the potential field involved which cancels the attraction towards the goal. The magnetic-field-inspired algorithm, on the other hand, does not encounter the same problem since the vector field employed is not repulsive.

The second scenario is where the environment is more complicated as it consists of many planar and spherical obstacles as shown in Fig. 6. Once again, we compare the performance of the electric-field-potential algorithm (Fig. 6a) and the magnetic-field-inspired algorithm (Fig. 6b) when applied to the kinematic model of the soft inflatable manipulator. In this scenario, we can see that when using the electric-field-potential, the robot does not get stuck as is the case in the previous environment. However, we can observe how the motion is less smooth compared to the motion when using the magnetic-field-inspired algorithm in Fig. $6 \mathrm{~b}$. This occurs especially in the final stage of the motion where we can observe that the obstacle causes more bending of the robot body in Fig. 6a compared to the robot in Fig. 6b whose shape does not get affected as much. This less smooth motion is caused by the repulsiveness of the vector field employed in Fig. 6a.

In the last scenario, the environment consists of more obstacles and the manipulator is expected to move in succession to several target points. This scenario mimics the situation when the manipulator needs to bring a sensor such as a cam- 


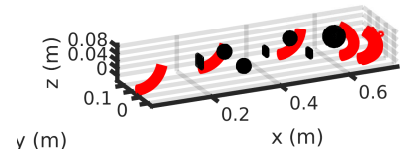

(a)

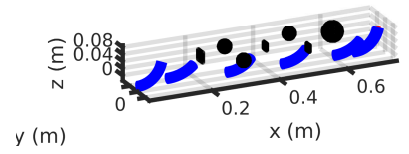

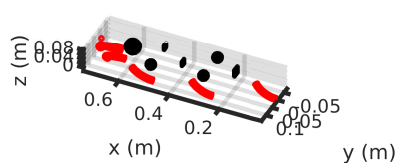

$\mathrm{y}(\mathrm{m})$

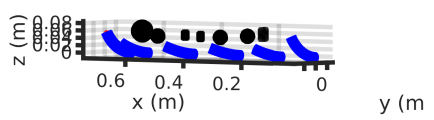

(b)

Fig. 6. The soft inflatable arm with a mobile base navigates an environment consisting of multiple obstacles viewed from two different perspectives in the left and the right. (a) The performance of an electric-field-potential algorithm and (b) a magnetic-fieldinspired algorithm are shown. The step-by-step configuration starts from left to right in the left pictures and vice versa in the right pictures.

era to inspect several locations in an unknown cluttered environment. In Fig. 7, we only show the manipulator's movement when using the proposed magneticfield-inspired navigation. To better visualise the manipulator's movement, we show the step-by-step motions in each figure from two different perspectives shown in Fig. 7a and Fig. 7b. In each figure, we can observe how magnetic-fieldinspired navigation is able to smoothly navigate the manipulator towards each of the target locations without colliding with multiple obstacles.

In all the scenarios presented from Fig. 5-Fig. 7, we can observe that the overall length of the manipulator does not vary too much during the course of the movement. This is a direct effect of the redundancy mechanism described in Section 3.3 which exploits the redundancy of the manipulator to avoid the actuating constraints when navigating towards the goal.

\section{Conclusions and Future Works}

In this paper, we have implemented magnetic-field-inspired navigation to guide the soft inflatable manipulator towards a target position while reactively avoiding collision with the environment. The beam-theory-based model is used to model the behaviour of the manipulator which uses a combined antagonistically tendon and pressure actuation. The redundancy in the manipulator combined with a planar mobile base is exploited to help the actuators stay under their maximum capability without affecting the manipulator's movement while navigating the environment. The navigation algorithm is shown to outperform the potentialfield-based navigation in its ability to smoothly and reactively avoid obstacles and reach the goal in simulation scenarios. Applying the algorithm to the real soft inflatable manipulator in a real-life environment while considering the dynamics of the system will be the focus of the future works. 

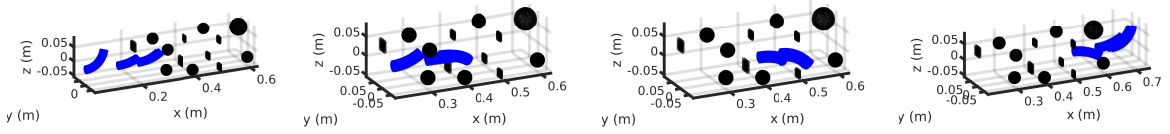

(a)
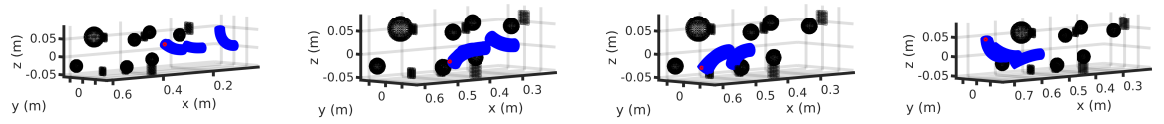

(b)

Fig. 7. The soft inflatable arm moves to several target points in succession in a cluttered environment viewed from two different perspectives in (a) and (b) using the magneticfield-inspired navigation.

\section{References}

1. Ataka, A., Lam, H.K., Althoefer, K.: Reactive magnetic-field-inspired navigation for non-holonomic mobile robots in unknown environments. In: Proc. IEEE Int. Conf. Robot. Autom. pp. 6983-6988 (May 2018). https://doi.org/10.1109/ICRA.2018.8463203

2. Ataka, A., Lam, H.K., Althoefer, K.: Reactive magnetic-field-inspired navigation method for robots in unknown convex 3-D environments. IEEE Robotics and Automation Letters 3(4), 3583-3590 (Oct 2018). https://doi.org/10.1109/LRA.2018.2853801

3. Ataka, A., Qi, P., Liu, H., Althoefer, K.: Real-time planner for multi-segment continuum manipulator in dynamic environments. In: Proc. IEEE Int. Conf. Robot. Autom. pp. 4080-4085 (May 2016)

4. Ataka, A., Shiva, A., Lam, H.K., Althoefer, K.: Magnetic-Field-Inspired Navigation for Soft Continuum Manipulator. In: 2018 IEEE/RSJ International Conference on Intelligent Robots and Systems (IROS). pp. 168-173 (Oct 2018). https://doi.org/10.1109/IROS.2018.8593592

5. Blumenschein, L.H., Gan, L.T., Fan, J.A., Okamura, A.M., Hawkes, E.W.: A Tip-Extending Soft Robot Enables Reconfigurable and Deployable Antennas. IEEE Robotics and Automation Letters 3(2), 949-956 (Apr 2018). https://doi.org/10.1109/LRA.2018.2793303

6. Chen, Y., Xu, W., Li, Z., Song, S., Lim, C.M., Wang, Y., Ren, H.: Safetyenhanced motion planning for flexible surgical manipulator using neural dynamics. IEEE Transactions on Control Systems Technology 25(5), 1711-1723 (Sep 2017). https://doi.org/10.1109/TCST.2016.2628806

7. Cianchetti, M., Ranzani, T., Gerboni, G., Nanayakkara, T., Althoefer, K., Dasgupta, P., Menciassi, A.: Soft robotics technologies to address shortcomings in today's minimally invasive surgery: the STIFF-FLOP approach. Soft robotics 1(2), $122-131(2014)$

8. Fras, J., Czarnowski, J., Macias, M., Glowka, J., Cianchetti, M., Menciassi, A.: New STIFF-FLOP module construction idea for improved actuation and sensing. In: Proc. IEEE Int. Conf. Robot. Autom. pp. 2901-2906 (May 2015). https://doi.org/10.1109/ICRA.2015.7139595 
9. Godage, I., Branson, D., Guglielmino, E., Caldwell, D.: Path planning for multisection continuum arms. In: Proc. Int. Conf. Mechatron. Autom. (ICMA). pp. 1208-1213 (Aug 2012). https://doi.org/10.1109/ICMA.2012.6283423

10. Granna, J., Godage, I.S., Wirz, R., Weaver, K.D., Webster, R.J., Burgner-Kahrs, J.: A 3-D volume coverage path planning algorithm with application to intracerebral hemorrhage evacuation. IEEE Robotics and Automation Letters 1(2), 876-883 (Jul 2016). https://doi.org/10.1109/LRA.2016.2528297

11. Hawkes, E.W., Blumenschein, L.H., Greer, J.D., Okamura, A.M.: A soft robot that navigates its environment through growth. Science Robotics 2(8) (2017). https://doi.org/10.1126/scirobotics.aan3028, http://robotics.sciencemag.org/content/2/8/eaan3028

12. Maghooa, F., Stilli, A., Noh, Y., Althoefer, K., Wurdemann, H.: Tendon and pressure actuation for a bio-inspired manipulator based on an antagonistic principle. In: Proc. IEEE Int. Conf. Robot. Autom. pp. 2556-2561 (May 2015). https://doi.org/10.1109/ICRA.2015.7139542

13. Neumann, M., Burgner-Kahrs, J.: Considerations for follow-the-leader motion of extensible tendon-driven continuum robots. In: Proc. IEEE Int. Conf. Robot. Autom. pp. 917-923 (May 2016). https://doi.org/10.1109/ICRA.2016.7487223

14. Qi, P., Liu, C., Ataka, A., Lam, H.K., Althoefer, K.: Kinematic Control of Continuum Manipulators Using a Fuzzy-Model-Based Approach. IEEE Transactions on Industrial Electronics 63(8), 5022-5035 (Aug 2016). https://doi.org/10.1109/TIE.2016.2554078

15. Qi, P., Qiu, C., Liu, H., Dai, J., Seneviratne, L., Althoefer, K.: A novel continuum-style robot with multilayer compliant modules. In: Proc. IEEE/RSJ Int. Conf. Intell. Robots Syst. pp. 3175-3180 (Sep 2014). https://doi.org/10.1109/IROS.2014.6943002

16. Rus, D., \& Tolley, M.T.: Design, fabrication and control of soft robots. Nature 521， 467-475 (2015). https://doi.org/doi:10.1038/nature14543, http://www.nature.com/nature/journal/v521/n7553/abs/nature14543.html

17. Sadati, S.M.H., Naghibi, S.E., Shiva, A., Walker, I.D., Althoefer, K., Nanayakkara, T.: Mechanics of Continuum Manipulators, a Comparative Study of Five Methods with Experiments. In: Gao, Y., Fallah, S., Jin, Y., Lekakou, C. (eds.) Towards Autonomous Robotic Systems. pp. 686-702. Springer International Publishing, Cham (2017)

18. Stilli, A., Kolokotronis, E., Fra, J., Ataka, A., Althoefer, K., Wurdemann, H.A.: Static Kinematics for an Antagonistically Actuated Robot Based on a Beam-Mechanics-Based Model. In: 2018 IEEE/RSJ International Conference on Intelligent Robots and Systems (IROS). pp. 6959-6964 (Oct 2018). https://doi.org/10.1109/IROS.2018.8593674

19. Torres, L., Kuntz, A., Gilbert, H., Swaney, P., Hendrick, R., Webster, R., Alterovitz, R.: A motion planning approach to automatic obstacle avoidance during concentric tube robot teleoperation. In: Proc. IEEE Int. Conf. Robot. Autom. pp. 2361-2367 (May 2015). https://doi.org/10.1109/ICRA.2015.7139513

20. Wang, H., Chen, J., Lau, H.Y.K., Ren, H.: Motion planning based on learning from demonstration for multiple-segment flexible soft robots actuated by electroactive polymers. IEEE Robotics and Automation Letters 1(1), 391-398 (Jan 2016). https://doi.org/10.1109/LRA.2016.2521384

21. Wu, K., Wu, L., Ren, H.: Motion planning of continuum tubular robots based on centerlines extracted from statistical atlas. In: Proc. IEEE/RSJ Int. Conf. Intell. Robot. Syst. pp. 5512-5517 (Sep 2015). https://doi.org/10.1109/IROS.2015.7354158 